\title{
Body Composition, Not Body Weight, Is Related to Cardiovascular Disease Risk Factors and Sex Hormone Levels in Men
}

Karen R. Segal, Andrea Dunaif, Bemard Gutin, Janine Albu, Asa Nyman, and F. Xavier Pi-Sunyer

Division of Pediatric Cardiology, Department of Pediatrics, and the Division of Endocrinology, Department of Medicine, Mount Sinai

School of Medicine, New York, New York 10029; and Department of Medicine, College of Physicians and Surgeons,

Columbia University at St. Luke's-Roosevelt Hospital Center, New York, New York 10025

\begin{abstract}
To clarify the independent relationships of obesity and overweight to cardiovascular disease risk factors and sex steroid levels, three age-matched groups of men were studied: (i) 8 normal weight men, $<15 \%$ body fat, by hydrostatic weighing: (ii) 16 overweight, obese men, $>25 \%$ body fat and $135-160 \%$ of ideal body weight (IBW); and (iii) 8 overweight, lean men, 135-160\% IBW, but < 15\% fat. Diastolic blood pressure was significantly greater for the obese (mean \pm SEM, $82 \pm 2 \mathrm{mmHg}$ ) than the normal $(71 \pm 2)$ and overweight lean $(72 \pm 2)$ groups, as were low density lipoprotein levels $(131 \pm 9$ vs. $98+11$ and $98+14 \mathrm{mg} / \mathrm{dl}$ ), the ratio of high density lipoprotein to total cholesterol $(0.207 \pm 0.01$ vs. $0.308 \pm 0.03$ and $0.302 \pm 0.03)$, fasting plasma insulin (22 \pm 3 vs. $12 \pm 1$ and $13 \pm 2 \mu \mathrm{U} / \mathrm{ml})$, and the estradiol/testosterone ratio $(0.076 \pm 0.01$ vs. $0.042 \pm 0.02$ and $0.052 \pm 0.02) ; P<0.05$. Estradiol was $25 \%$ greater for the overweight lean group $(40 \pm 5 \mathrm{pg} / \mathrm{ml})$ than the obese $(30 \pm 3$ $\mathrm{pg} / \mathrm{ml}$ ) and normal groups $(29 \pm 2 \mathrm{pg} / \mathrm{ml}), P=0.08$, whereas total testosterone was significantly lower in the obese $(499 \pm 33$ ng/dl) compared with the normal and overweight, lean groups (759 \pm 98 and $797 \pm 82 \mathrm{ng} / \mathrm{dl})$. Estradiol was uncorrelated with risk factors and the estradiol/testosterone ratio appeared to be a function of the reduced testosterone levels in obesity, not independently correlated with lipid levels after adjustment for body fat content. Furthermore, no risk factors were signifcantly different between the normal and overweight lean groups. We conclude that $(a)$ body composition, rather than body weight per se, is associated with increased cardiovascular disease risk factors; and $(b)$ sex steroid alterations are related to body composition and are not an independent cardiovascular disease risk factor.
\end{abstract}

\section{Introduction}

The association between obesity and increased risk for acquiring cardiovascular disease has been well established (1-3), particularly by recent data from the Framingham study, in which obesity was shown to be a significant independent predictor for

This paper was presented in part at the 59th Scientific Sessions of the American Heart Association, November 1986, Dallas, TX 75231.

Address correspondence and reprint requests to Dr. Segal, Box 1201, Div. of Pediatric Cardiology, Mount Sinai School of Medicine, 1 Gustave Levy Place, New York, NY 10029.

Received for publication 12 January 1987 and in revised form 22 April 1987.

\section{J. Clin. Invest.}

(c) The American Society for Clinical Investigation, Inc. 0021-9738/87/10/1050/06 $\$ 2.00$

Volume 80, October 1987, 1050-1055 cardiovascular disease (4). However, there are several related issues that remain unclear. One is the specific relationship of obesity vs. being overweight to cardiovascular disease risk. The National Health and Nutrition Examination Survey (NHANES I, 1971 to 1974) suggested that men who were overweight, determined by relative weight for height, but muscular and not obese, according to triceps skinfold thickness, had greater risk for acquiring cardiovascular disease than normal weight men (5-7). In the National Health and Nutrition Examination Survey population, men were cross-classified according to percentile ranking on body mass index (BMI) ${ }^{1}$ and sum of the triceps and subscapular skinfold thicknesses into the following categories: $(a)$ overweight and obese; $(b)$ overweight and not obese; $(c)$ not overweight and obese; and $(d)$ not overweight and not obese. The incidences of diastolic hypertension $(>95 \mathrm{mmHg})$ and hypercholesterolemia were 2.5 and 1.7 times greater, respectively, in men who were overweight and not obese than in normal weight nonobese men (7). Other studies have also proposed that a large body build or muscular somatotype are associated with increased risk for premature coronary disease $(8,9)$. However, previous investigations of cardiovascular disease risk factors in relation to overweight and obesity have lacked rigorous assessment of body composition by an established technique such as hydrodensitometry, total body water, or total body potassium.

Another aspect of cardiovascular disease risk that has received considerable investigative attention is the relationship of sex steroids to lipid and lipoprotein levels and other risk factors. Recent studies have proposed that in men, hyperestrogenemia may be a predisposing factor for coronary disease and that an increased ratio of estradiol to testosterone may be related to the expression of cardiovascular disease risk factors $(10,11)$. Obese men have elevated estrogen levels (12), which have been attributed to an enlarged adipose tissue mass that is a site for conversion of androgens to estrogens (13). Lindholm et al. (14) have suggested that the association between coronary heart disease and hyperestrogenemia might be mediated by obesity, since after adjustment for the correlation between estradiol levels and body weight no relationships were observed between sex hormone levels and cardiovascular disease risk factors. In contrast, other investigators (15) have reported that estrone but not estradiol levels were elevated in patients who survived myocardial infarction, compared with controls, and concluded that the abnormal hormone levels were not related to obesity in view of the fact that neither the patients nor the control were $>20 \%$ above desirable weight. Body composition was not measured in either of these two studies.

1. Abbreviations used in this paper: BMI, body mass index; LBM, lean body mass; OGTT, oral glucose tolerance test; SHBG, sex-hormone binding globulin. 
Thus, the interactions among obesity, being overweight, sex steroids, and risk factors remain unclear and warrant further investigation.

The present study was designed to clarify the independent relationships among body composition, sex steroids, and cardiovascular disease risk factors in men. This was accomplished by use of a model in which the putative relationship between overweight and obesity was uncoupled.

\section{Methods}

Subjects. 32 men between the ages of 20 and 35 participated in this study. Group A $(n=16)$ was overweight, which was defined as a BMI between 29 and 35 (16), and obese, which was defined as hydrostatically determined body fat (see below) $>5 \%$. Group B $(n=8)$ was overweight, matched with group A with respect to total body weight and BMI, but lean, defined as $<15 \%$ body fat. Group $C(n=8)$ was normal weight and lean, with a BMI of 20 to 22 , and had $<15 \%$ body fat. Groups $\mathrm{A}$ and $\mathrm{C}$ were matched with respect to lean body mass (LBM), determined by hydrostatic weighing. All three groups were matched with respect to age and height. All subjects were healthy, with no personal or family history of cardiovascular disease, diabetes mellitus, or other metabolic disease. An oral glucose tolerance test was administered (see below) to ensure that all subjects were nondiabetic and had normal glucose tolerance, according to the criteria of the National Diabetes Data Group (17). To control for a possible intervening effect of level of cardiorespiratory fitness, aerobically trained men were not accepted into the study. None of the men engaged in regular aerobic exercise, but some of the men, particularly in group B, took part in weight lifting, or primarily anaerobic sports such as wrestling, football, or power lifting, which do not significantly increase cardiorespiratory fitness (18). All subjects were weight-stable at the time of the study with no more than a 2-kg weight loss or gain over the 6 mo before the study. The subjects were instructed to refrain from any physical exercise and alchohol consumption and to consume a weight-maintenance diet containing at least $250 \mathrm{~g}$ carbohydrate per day for at least $3 \mathrm{~d}$ before blood sampling. Before acceptance into the study a dietary history was obtained from each subject. To control for possible confounding effects of diet on metabolic and hormonal parameters, men who engaged in unusual dietary practices (vegetarianism, high or low protein diets, amino acid or vitamin supplementation, or fasting) were excluded from the study. The men were nonsmokers and were not taking any medications. The subjects were screened for use of anabolic, androgenic drugs by interview, physical examination, and plasma steroid analyses. For the men who were accepted into the study, there was no clinical or biochemical evidence of exogenous steroid use. The written informed consent was obtained from all subjects and the protocol was approved by the Institutional Review Board of the St. Luke's-Roosevelt Institute for Health Sciences.

Densitometry. Body fat content and LBM were determined by densitometry. The subjects were tested in the morning after a 12-h fast. Body density was determined by hydrostatic weighing in a stainless steel tank in which a swing seat was suspended from a Chatillon 15-kg scale. The subjects submerged beneath the surface of the water while expiring maximally and remained as motionless as possible at the point of maximal expiration for roughly $5 \mathrm{~s}$, while underwater weight was recorded. After several practice trials to familiarize the subjects with the test procedure, 10 trials were performed. The estimated underwater weight was the highest value, and this value was reproduced three times (19). Residual lung volume was estimated by means of the closed-circuit oxygen dilution method of Wilmore (20), with use of a 9-liter spirometer (Warren E. Collins, Braintree, MA) and a nitrogen analyzer (Fiske Med-Science, St. Louis, MO). Two trials were performed while the subjects assumed a sitting position that duplicated body position in the tank during underwater weighing. Body density was calculated from the formula of Goldman and Buskirk (21) and percent body fat was derived from body density by use of the Siri equation (22):

percent body fat $=4.95 /$ density -4.5 .

LBM is the difference between total body weight and fat weight, where

fat weight $=$ total body weight $\times$ percent body fat.

Blood sampling and assay procedures. A fasting venous blood sample was obtained in the morning after an overnight $(12 \mathrm{~h})$ fast from an antecubital vein. Plasma estradiol and testosterone were measured by radioimmunoassay after diethyl ether extraction and Celite chromatography, with minor modifications of previously reported procedures $(23,24)$. Sex-hormone binding globulin (SHBG) was determined by ammonium sulfate precipitation according to the method of Rosner (25). The percentage of non-SHBG-bound (free and albumin bound) testosterone was determined by the method of Tremblay and Dube (26), with the exception that the incubation with labeled steroid was performed at $37^{\circ} \mathrm{C}$, instead of at room temperature. The amount of non-SHBG-bound testosterone was calculated by multiplying the total testosterone level by the percentage of non-SHBG-bound testosterone.

Total cholesterol and high density lipoprotein (HDL) cholesterol were measured enzymatically according to the procedure described by Allain et al. (27). Triglycerides were assayed enzymatically based on the ultraviolet procedure of Buculo and David (28). Low density lipoprotein (LDL) cholesterol levels were calculated with use of the following formula (29): $\mathrm{LDL}=$ total cholesterol $-(\mathrm{HDL}+$ triglycerides $\div 5$ ).

Oral glucose tolerance test (OGTT). After a fasting blood sample was drawn, a 75-g oral glucose load (Custom Laboratories, Inc., Baltimore, $M D$ ) was given and venous blood samples were drawn at $30-\mathrm{min}$ intervals for $2 \mathrm{~h}$. The plasma was separated and analyzed for glucose and insulin. A glucose analyzer (Beckman Instrument Inc., Fullerton, CA) was used for measuring plasma glucose (30). Plasma insulin was measured by radioimmunoassay with charcoal absorption with use of a human insulin standard (31). The integrated areas under the 2-h glucose and insulin curves were calculated.

Blood pressure determination. Resting blood pressure was measured three times with use of an aneroid sphygmomanometer while the subjects were seated quietly. The same investigator made all blood pressure measurements.

Aerobic fitness test. Maximal aerobic fitness $\left(\mathrm{VO}_{2} \mathrm{max}\right)$ was determined by a continuous multistage exercise test on a cycle ergometer (Monark-Crescent Ab, Varbeg, Sweden). Before the test, time was allotted for the subjects to become familiar with cycling on an ergometer at a constant pedalling rate and to breathing through the apparatus used for metabolic measurements. The subjects began cycling at a rate of $50 \mathrm{rpm}$ with zero external resistance (unloaded cycling). A metronome was used to assist the subject in maintaining the proper pedalling rate. The work rate was increased in $25-\mathrm{W}$ increments every $2 \mathrm{~min}$ until volitional exhaustion was reached and the subject refused to continue despite vocal encouragement or until he was unable to maintain the pedalling rate. Ventilatory measurements were made continuously by open-circuit respirometry with use of a Horizon Metabolic Measurement Cart, which includes a turbine volume transducer, an OM-1 1 polarographic oxygen $\left(\mathrm{O}_{2}\right)$ analyzer, and an LB-2 non-dispersive infrared carbon dioxide $\left(\mathrm{CO}_{2}\right)$ analyzer. The subjects breathed through a non-rebreathing valve (Hans Rudolph, Inc., Kansas City, MO) and used mouthpieces and noseclips. The gas analyzers were calibrated before and after each aerobic fitness test with $100 \%$ nitrogen, room air, and a gas mixture containing $4 \% \mathrm{CO}_{2}$ and $16 \% \mathrm{O}_{2}$. For each measurement, oxygen consumption, carbon dioxide production, and minute ventilation were obtained.

Analysis of data. One-way analyses of variance were applied to the data to determine the significance of differences among the three groups in physical characteristics, sex hormones, lipid and lipoprotein levels, the OGTT results, aerobic fitness, and blood pressure levels. Significant $F$ ratios were followed by posthoc comparisons, with use of the Duncan's range procedure, to determine the source of differences 
among groups (32). Pearson product-moment correlations were calculated among the lipid, hormonal, risk factor, and body composition parameters. The similarity of the correlations and regression coefficients obtained within each individual group were tested statistically before pooling the data from the three groups to ensure that the assumption of homogeneity of regression across groups was met (32). Partial correlation analyses were applied to determine the independent relationships among risk factors, sex hormones, and body composition, as described in the Results section.

For all statistical analyses the 0.05 level of significance was used.

\section{Results}

As shown in Table I, the three groups of men were similar with respect to age and height. The obese men (group A) were almost threefold fatter than the other two groups but they were matched to group C with respect to LBM. LBM was roughly $20 \mathrm{~kg}$ greater in group B than in the other two groups. Maximal oxygen consumption was similar for the three groups except when expressed relative to total body weight, in which case it was significantly greater for group $C$ than for the two groups of overweight men. Systolic blood pressure was not different among the three groups, but diastolic blood pressure was significantly greater for the obese men than for the other two groups.

The sex steroid data are shown in Table II. Testosterone was lower and the estradiol/testosterone ratio was significantly higher for the obese men than in the other two groups. Estradiol was higher for group B than for the other two groups, but this difference did not achieve statistical significance $(P$ $=0.08$ ). SHBG was significantly lower in the obese group than in the two lean groups. The percentage of non-SHBG-bound testosterone was significantly greater for the obese men than for the two groups of lean men. The calculated amount of non-SHBG-bound testosterone was not significantly different among the three groups. Estradiol was significantly correlated with LBM $(r=0.39, P<0.05)$ but was unrelated to body fat content $(r=-0.09)$. Body fat content was strongly correlated with total testosterone $(r=-0.62, P<0.01)$, SHBG $(r=$ $-0.37, P<0.05)$, non-SHBG-bound testosterone $(r=0.45, P$ $<0.05)$, and estradiol/testosterone $(r=0.50, P<0.01)$.

Fasting plasma glucose was not different among the three groups, but fasting plasma insulin and the areas under the 2-h glucose and insulin curves were significantly higher for the obese men compared with the two lean groups (see Table III). As shown in Table IV, the three groups did not differ significantly with respect to triglycerides, total cholesterol, and HDL. Perhaps with larger sample sizes the differences among groups in triglycerides, total cholesterol, and $\mathrm{HDL}$ would have achieved statistical significance. The power $(1-\beta)$ to detect differences among groups at the $\alpha=0.05$ level of significance was 0.60 for triglycerides, 0.65 for cholesterol, and 0.75 for HDL. However, the ratio of HDL/total cholesterol was signifcantly lower and LDL was greater in the obese group compared with the two lean groups. Body fat was significantly correlated with systolic blood pressure $(r=0.60, P<0.01)$, plasma insulin $(r=0.74, P<0.01)$, LDL $(r=0.56, P<0.01)$, total cholesterol $(r=0.53, P<0.01)$, and HDL/total cholesterol $(r=-0.63, P<0.01)$. LBM and total body weight were uncorrelated with any of these parameters.

Apparent correlations were observed between the estradiol/testosterone ratio and both LDL $(r=0.34, P<0.05)$ and total cholesterol $(r=0.37, P<0.05)$. To determine the relationship between estradiol/testosterone and lipids, independent of the influence of obesity, partial correlations between estradiol/testosterone and lipids, controlling for body fat, were calculated (32). When body fat was partialled out statistically, the correlations of estradiol/testosterone with cholesterol $(r$ $=0.14)$ and with LDL $(r=0.08)$ were no longer significant. On the other hand, when the effect of estradiol/testosterone was controlled for by partial correlation, significant correlations persisted between body fat and cholesterol $(r=0.43, p$ $<0.05)$, LDL $(r=0.48, P<0.05)$, HDL $(r=0.39, P<0.05)$, and HDL/total cholesterol $(r=0.63, P<0.01)$.

\section{Discussion}

In the present study we made use of a new model in which LBM and total body weight were uncoupled from their usual positive correlation with body fat; in addition to a nonoverweight, lean control group we also studied an overweight, lean control group. While this latter group was matched to the obese men with respect to total body weight and BMI, an elevated LBM accounted for the excess weight of the men in group B, whereas adipose tissue accounted for the excess weight in the obese group. Furthermore, groups $A$ and $C$ were matched with respect to LBM, thus eliminating the possible

Table I. Characteristics of Overweight and Obese (Group A), Overweight but Lean (Group B), and Normal Weight, Lean Men (Group C)*

\begin{tabular}{|c|c|c|c|c|}
\hline & Group A & Group B & Group C & $P$ value \\
\hline Age & $26 \pm 1$ & $25 \pm 1$ & $24 \pm 2$ & NS \\
\hline Weight $(k g)$ & $\underline{94 \pm 3}$ & $95 \pm 4^{\ddagger}$ & $74 \pm 2$ & $P<0.001$ \\
\hline Height $(\mathrm{cm})$ & $178 \pm 2$ & $181 \pm 3$ & $179 \pm 3$ & NS \\
\hline BMI $\left(k g / m^{2}\right)$ & $\underline{30 \pm 1}$ & $30 \pm 1$ & $23 \pm 1$ & $P<0.001$ \\
\hline Percent body fat & $30 \pm 1$ & $\underline{10 \pm 1}$ & $12 \pm 1$ & $P<0.001$ \\
\hline $\operatorname{LBM}(k g)$ & $\underline{66 \pm 2}$ & $85 \pm 3$ & $\underline{65 \pm 2}$ & $P<0.001$ \\
\hline $\mathrm{VO}_{2} \max (\mathrm{ml} / \mathrm{min})$ & $2,920 \pm 137$ & $3,341 \pm 85$ & $3,387 \pm 240$ & NS \\
\hline$(\mathrm{ml} / \mathrm{kg} / \mathrm{min})$ & $\underline{32 \pm 2}$ & $36 \pm 1$ & $45 \pm 3$ & $P<0.001$ \\
\hline Systolic blood pressure $(\mathrm{mmHg})$ & $123 \pm 3$ & $119 \pm 4$ & $117 \pm 2$ & NS \\
\hline Diastolic blood pressure $(\mathrm{mmHg})$ & $82 \pm 2$ & $\underline{72 \pm 2}$ & $71 \pm 2$ & $P<0.05$ \\
\hline
\end{tabular}

\footnotetext{
*Values are mean \pm SEM. ${ }^{\ddagger}$ Groups sharing the same underline do not differ significantly.
} 
Table II. Sex Hormone Levels in Overweight and Obese (Group A), Overweight but Lean (Group B), and Normal Weight, Lean Men (Group C)*

\begin{tabular}{lcccc}
\hline & Group A & Group B & Group C & $P$ value \\
\hline Testosterone $(n g / d l)^{\ddagger}$ & $499 \pm 33$ & $\frac{797 \pm 82}{40 \pm 5}$ & $759 \pm 98^{\S}$ & $29 \pm 2$ \\
Estradiol $(p g / m l)^{\ddagger}$ & $30 \pm 3$ & $\underline{0.052 \pm 0.02}$ & $0.042 \pm 0.01$ & $P<0.05$ \\
Estradiol/testosterone & $0.076 \pm 0.01$ & $\underline{0.70 \pm 0.05}$ & $0.69 \pm 0.05$ & $P<0.05$ \\
SHBG $(\mu g / d l)$ & $0.57 \pm 0.03$ & $\underline{47 \pm 3}$ & $41 \pm 3$ & $P<0.05$ \\
Non-SHBG-bound testosterone & & $370 \pm 34$ & $311 \pm 53$ & NS \\
$\quad$ Percent & $55 \pm 3$ & & &
\end{tabular}

* Values are mean \pm SEM. ${ }^{\ddagger}$ To convert to nanomoles per liter, multiply estradiol by 0.0037 , and testosterone and non-SHBG-bound testosterone by 0.0346 . Groups sharing the same underline do not differ significantly.

effect of the well-documented elevated LBM in obesity $(33,34)$ on metabolic and hormonal parameters.

Cardiovascular disease risk factors in relation to overweight and obesity. Diastolic blood pressure, fasting plasma insulin and the insulin response to an oral glucose load, and LDL were significantly greater while HDL/total cholesterol was significantly lower for the obese men compared with the two lean groups. These findings support previous reports that obesity constitutes an independent risk factor for cardiovascular disease (1-4). However, no risk factors were found to be significantly different between the lean, overweight men (group B) and the normal weight, lean men. Previous suggestions of adverse health implications of being overweight and nonobese might be attributed to lack of a rigorous method for assessing adiposity. For example, as Van Itallie has pointed out $(7,35)$ in the National Center for Health Statistics report $(36)$, in which hypertension and hypercholesterolemia were reported to be significantly more prevalent in overweight, nonobese individuals than in nonoverweight individuals, obesity was determined by the sum of the triceps and subscapular skinfold thicknesses, and it is possible that some individuals whose excess fat was located primarily in other areas of the body (abdominal, femoral, or gluteal regions), or located primarily internally compared with subcutaneously, were misclassified. Furthermore, recent investigations have shown that the pattern of body fat distribution has important health implications; abdominal accumulation of body fat is associated with elevated glucose, insulin, triglycerides, and blood pressure $(37,38)$. In the absence of whole-body composition assessment, this effect of body fat distribution on cardiovascular disease risk could have been masked by categorization of obesity based on triceps and subscapular skinfolds. Thus, misclas- sification of subjects and variation in the pattern of body fat distribution may explain, in part, the relatively greater frequency of hypertension and hyperlipidemia among the overweight, nonobese individuals. Other reports of an association between elevated muscularity or body build and risk of premature coronary heart disease are based only on assessment of somatotype or body frame size, with no control or quantitative determination of body fat mass $(8,9)$. The present study fails to show any association between elevated LBM or being overweight per se, independent of obesity, and blood pressure, insulin, or lipid levels.

Sex steroids and body composition. The reduced total testosterone and SHBG levels, and higher percentage of nonSHBG-bound testosterone observed in the obese men compared with the two lean groups confirm previous studies (12, 13). The non-SHBG-bound testosterone, which includes both free testosterone and albumin-bound testosterone, represents the biologically available testosterone better than free testosterone alone or total testosterone $(12,13)$. The half-dissociation time of testosterone from SHBG is relatively slow, so that it is biologically unavailable to target tissues, whereas albumin-bound and free testosterone are largely biologically available to tissues and are physiologically active. Biologically available (free and albumin bound) testosterone levels remain unchanged by obesity, however, because of a concomitant decrease in SHBG levels (39). The lower testosterone levels in obese men have been attributed in part to conversion of androgens to estrogens in adipose tissue $(13,40,41)$. Elevated estradiol levels have also been reported in obese men, but only when the men exceeded roughly $160 \%$ of desirable body weight, according to the Metropolitan Life Insurance weightfor-height tables (12). Kley et al. (40) found that conversion of

Table III. Oral Glucose Tolerance Test Results in Overweight and Obese (Group A), Overweight but Lean (Group B), and Normal Weight, Lean Men (Group C)*

\begin{tabular}{|c|c|c|c|c|}
\hline & Group A & Group B & Group C & $P$ value \\
\hline Fasting insulin $(\mu U / m l)^{\ddagger}$ & $22 \pm 3$ & $\underline{13 \pm 2}$ & $12 \pm 1^{* \|}$ & $P<0.05$ \\
\hline Fasting glucose $(m g / d l)^{\ddagger}$ & $86 \pm 2$ & $84 \pm 2$ & $88 \pm 2$ & NS \\
\hline Insulin area & $444 \pm 18$ & $\underline{216 \pm 33}$ & $265 \pm 58$ & $P<0.05$ \\
\hline Glucose area & $474 \pm 18$ & $402 \pm 19$ & $399 \pm 23$ & $P<0.05$ \\
\hline
\end{tabular}

* Values are mean+SEM. ${ }^{\ddagger}$ To convert insulin to picomoles per liter multiply by 7.175 ; to convert glucose values to millimoles per liter multiply by 0.0625 . Integrated over $2 \mathrm{~h}$ after a $75-\mathrm{g}$ oral glucose load. "Groups sharing the same underline do not differ significantly. 
Table IV. Lipid and Lipoprotein Levels in Overweight and Obese (Group A), Overweight but Lean (Group B), and Normal Weight, Lean Men (Group C)*

\begin{tabular}{lcccc}
\hline & Group A & Group B & Group C & P value \\
\hline Cholesterol $(m g / d l)^{\ddagger}$ & $196 \pm 9$ & $164 \pm 18$ & $167 \pm 13$ & NS \\
HDL $(m g / d l)$ & $40 \pm 3$ & $49 \pm 6$ & $49 \pm 2$ & NS \\
HDL $/$ cholesterol & $0.207 \pm 0.01$ & $\frac{0.302 \pm 0.03}{98 \pm 14}$ & $0.308 \pm 0.03^{8}$ & $98 \pm 11$ \\
LDL $(m g / d l)$ & $131 \pm 9$ & $\left.\frac{98 \pm 10}{89}\right)$ & $99 \pm 23$ & $P<0.05$ \\
Triglycerides $(m g / d l)^{\ddagger}$ & $125 \pm 19$ & & NS
\end{tabular}

* Values are mean \pm SEM. $\quad{ }^{\ddagger}$ To convert to millimoles per liter multiply cholesterol by 0.02586 and triglycerides by $0.01129 . \quad{ }^{\S}$ Groups sharing the same underline do not differ significantly.

androstenedione to estrone and estradiol was significantly greater in men who were $>160 \%$ of desirable weight than in men who were $90-120 \%$ of desirable weight, and concluded that aromatization of androgens in the increased adipose tissue mass accounts for the elevated estrogen levels in obese men. This conclusion is supported by in vitro and in vivo studies of the conversion of androgens to estrogens in fat tissue $(41,42)$. However, the possibility that aromatization of androgens to estrogens can occur in other tissues, specifically, in muscle, cannot be ruled out from the studies of Kley et al. $(12,40,43)$, owing to the intercorrelation between muscle mass and body fat mass, and the fact that LBM increases with increasing obesity $(33,34)$. In other words, previous investigations have drawn conclusions about the conversion of androgens to estrogens in adipose tissue from correlations with indices of obesity without regard to the intercorrelations among body composition parameters. In the present study, an experimental model was used in which body composition parameters were uncorrelated: the correlation between lean mass and fat mass was not significant $(r=-0.26)$.

In the present study, no difference in estradiol levels between obese men and lean men of similar LBM was observed. Conversely, estradiol levels were $25-30 \%$ greater in the overly muscular group compared with the other two groups, and estradiol was significantly correlated with LBM $(P<0.05)$, but not with body fat mass. This supports, indirectly, the finding of Longcope et al. (42) based on human forearm perfusion studies, that muscle may be a significant site for conversion of androgens to estrogens, accounting for roughly $25 \%$ of the total extragonadal aromatization of androgens to estrogens. The overweight men in the present study were roughly $135-165 \%$ of desirable weight. The degree of overweight in the subjects was constrained by the necessity of matching lean and obese men with respect to total body weight and degree of overweight, and matching the obese and normal weight men with respect to LBM. We were unable to recruit men who were $<15 \%$ fat but $>165 \%$ of desirable weight and who met the other criteria for inclusion in the study. Perhaps if the lean overweight men had been even more overweight the elevation in estradiol levels would have achieved statistical significance, in view of the fact that there appears to be a threshold in overweight before estradiol levels become abnormally high (43). Similarly, the obese men probably did not demonstrate significantly increased estradiol levels because they were not as fat as the men in previous studies, and therefore their body fat mass and LBM were not as high as the levels encountered in more severe obesity.
Sex steroids in relation to cardiovascular disease risk factors. The relationship between sex hormones and cardiovascular disease risk factors is controversial. According to the hypothesis put forth by Phillips (10), hyperestrogenemia in men is significantly linked with coronary disease, and an elevated estradiol/testosterone ratio may provoke the expression of cardiovascular disease risk factors. In contrast, a recent investigation (44) of sex hormones and lipoproteins in men demonstrated significant negative correlations between estradiol and both LDL $(r=-0.52)$ and total cholesterol $(r=-0.56)$. It is possible that an intervening effect of body fat may explain these discrepant observations. In the present study, apparent correlations of the estradiol/testosterone ratio to LDL and total cholesterol do not reflect a relationship between hyperestrogenemia and lipids, but are a reflection of the reduced testosterone levels in obese men. Significant correlations of the estradiol/testosterone ratio to LDL and total cholesterol were eliminated when the influence of body fat was suppressed. This supports the observation by Lindholm et al. (14) that no significant relationships existed between sex hormone levels and lipid levels after adjustment for age and body weight. However, body fatness was not measured in that study (14).

Thus, total body weight per se is not independently related to either sex hormone levels or cardiovascular disease risk factors, whereas the individual body composition parameters (fat mass and lean mass) are significant determinants of both. In conclusion, the results of this study, in which a novel approach was taken to uncouple obesity from body weight and LBM, demonstrate that excess adiposity, but not elevated total body weight, is independently related to cardiovascular disease factors. Furthermore, the documented hyperestrogenemia in obesity may be due to the increase in LBM associated with obesity as well as to the increase in adipose tissue, and may be related to the extragonadal aromatization of androgens to estrogens in muscle as well as in fat tissue. Independent from the effect of obesity on total testosterone, the estradiol/testosterone ratio is uncorrelated with cardiovascular disease risk factors.

\section{Acknowledgments}

This study was supported in part by grants from the National Institutes of Arthritis, Diabetes, and Digestive and Kidney Diseases (DK37665 and DK26687).

\section{References}

1. Bottiger, L.E., and L.A. Carson. 1980. Risk factors for ischemic vascular death for men in the Stockholm Prospective Study. Atherosclerosis. 36:389-408. 
2. Larsson, B., P. Bjorntorp, and G. Tibblin. 1981. The health consequences of moderate obesity. Int. J. Obesity. 5:97-116.

3. Rabkin, S. W., F. A. L. Mathewson, and P. H. Hsu. 1977. Relation of body weight to development of ischemic heart disease in a chohort of young North American men after a 26-year observation period: The Manitoba Study. Am. J. Cardiol. 39:452-458.

4. Hubert, H. B., M. Feinleib, P. M. McNamara, and W. P. Castelli. 1983. Obesity as an independent risk factor for cardiovascular disease: a 26-year follow-up of participants in the Framingham Heart Study. Circulation. 67:968-977.

5. Roberts, J., and M. Rowland. 1981. Hypertension in adults 25-74 years of age, United States, 1971-1975. National Center for Health Statistics, Washington, D.C. DHHS publication No. (PHS)811671, Vital and Health Statistics, series 11, No. 221.

6. Abraham, S., M. Carroll, and M. F. Najjar. 1978. Total serum cholesterol levels for adults 18-74 years. U.S. Government Printing Office, Washington, D.C. DHEW publication No. (PHS)78-1652, Vital and Health Statistics, series 11, No. 205.

7. Van Itallie, T. B. 1985. Health implications of overweight and obesity in the United States. Ann. Intern. Med. 103:983-988.

8. Rissanen, V. 1975. Coronary and aortic atherosclerosis in relation to body-build factors. Ann. Clin. Res. 7:402-411.

9. Spain, D. M., V. A. Broders, and J. Huss. 1953. Observations of atherosclerosis of coronary arteries in males under age 46: necropsy study with special reference to somatotypes. Ann. Intern. Med. 38:254-277.

10. Phillips, G. B. 1978. Sex hormones, risk factors and cardiovascular disease. Am. J. Med. 65:7-11.

11. Phillips, G. B., W. P. Castelli, R. D. Abbott, and P. M. McNamara. 1983. Association of hyperestrogenemia and coronary heart disease in men in the Framingham cohort. Am. J. Med. 74:863-869.

12. Kley, H. K., H. G. Solbach, J. C. McKinnan, and J. L. Kruskemper. 1979. Testosterone decrease and estrogen increase in male patients with obesity. Acta Endocrinol. 91:553-563.

13. Schneider, G., M. A. Kirschner, R. Berkiwitz, and N. H. Ertel. 1979. Increased estrogen production in obese men. J. Clin. Endocrinol. Metab. 48:633-637.

14. Lindholm, J., P. Winkel, U. Brodthagen, and F. Gyntelberg. 1982. Coronary risk factors and plasma sex hormones. Am. J. Med. 73:648-651.

15. Zumoff, B., R. G. Troxler, and J. O'Connor. 1982. Abnormal hormone levels in men with coronary artery disease. Arteriosclerosis. 2:58-67.

16. Bray, G. A. 1978. Definition, measurement, and classification of the syndromes of obesity. Int. J. Obesity 2:99-112.

17. National Diabetes Data Group. 1979. Classification and diagnosis of diabetes mellitus and other categories of glucose tolerance. Diabetes. 28:1039-1057.

18. McArdle, W. D., F. I. Katch, and V. I. Katch. 1981. Exercise Physiology: Energy, Nutrition, and Human Performance. Lea and Febiger, Philadelphia. 80-117.

19. Behnke, A. R., and J. H. Wilmore. 1974. Evaluation of Body Build and Composition. Prentice-Hall Inc., Englewood Cliffs, NJ. $100-120$.

20. Wilmore, J. H. 1969. A simplified method for determination of residual lung volume. J. Appl. Physiol. 27:96-100.

21. Goldman, R. F., and E. R. Buskirk. 1961. Body volume measurement by underwater weighing: description of a method. In Techniques for Measuring Body Composition. J. Brozek, and A. Henschel, editors. National Academy of Sciences, Washington, D.C. 78-89.

22. Siri, W. E. 1961. Body composition from fluid spaces and density: analysis of methods. In Techniques for Measuring Body Composition. J. Brozek and A. Henschel, editors. National Academy of Sciences, Washington, D.C. 223-244.

23. Nagai, N., and C. L. Longcope. 1971. Estradiol-17-beta and estrone: studies on their binding to rabbit uterine cytosol and their concentration in plasma. Steroids. 17:631-670.
24. Samojlik, E., M. A. Kirschnew, D. Silber, G. Schneider, and N. H. Ertel. 1984. Elevated production and metabolic clearance rates of androgens in morbidly obese women. J. Clin. Endocrinol. Metab. 59:949-956.

25. Rosner, W. 1972. A simplified method for the quantitative determination of testosterone-estradiol-binding globulin activity in human plasma. J. Clin. Endocrinol. Metab. 34:983-988.

26. Tremblay, R. R., and J. Y. Dube. 1974. Plasma concentrations of free and non-TeBG bound testosterone in women on oral contraceptives. Contraception. 10:599-603.

27. Allain, C. A., L. S. Poon, C. S. G. Chan, W. Richmond, and P. C. Fu. 1974. Enzymatic determination of total serum cholesterol. Clin. Chem. 20:470-477.

28. Buculo, G., and H. David. 1973. Quantitative determination of serum triglycerides by use of enzymes. Clin. Chem. 19:475-482.

29. Lipid Research Clinics Program. 1974. Manual of Laboratory Operations: Lipid and Lipoprotein Analysis. National Heart and Lung Institute, National Institutes of Health, Bethesda, MD. DHEW publication No. (NIH)75-620.

30. Hugget, A. S., and D. A. Nixon. 1957. Use of glucose oxidase peroxidase, and O-dianisidine in determination of blood and urinary glucose. Lancet. ii:368-370.

31. Herbert, V., K. S. Lau, C. W. Gottlieb, and S. J. Bleicher. 1965. Coated charcoal immunoassay of insulin. J. Clin. Endocrinol. Metab. 25:1375-1384.

32. Winer, B. J. 1971. Statistical Principles in Experimental Design. 2nd ed. McGraw-Hill Inc., New York. 258-310.

33. Forbes, G. B., and S. L. Welle. 1982. Lean body mass in obesity. Int. J. Obesity. 7:1073-1079.

34. James, W. P. T., K. Bailes, H. L. Davies, and M. J. Dauncey. 1978. Elevated metabolic rates in obesity. Lancet. i:1122-1125.

35. Van Itallie, T. B., and S. Abraham. 1985. Some hazards of obesity and its treatment. In Recent Advances in Obesity Research: IV. J. Hirsch and T. B. Van Itallie, editors. John Libbey \& Co. Ltd., London. 1-19.

36. Abraham, S., M. Carroll, M. F. Najjar, and R. Fulwood. 1983. Obese and overweight adults in the United States. National Center for Health Statistics, Hyattsville, MD. DHHS publication No. 83-1680, Vital and Health Statistics, series 11, No. 230.

37. Bjorntorp, P. 1985. Adipose tissues in obesity. In Recent Advances in Obesity Research: IV. J. Hirsch and T. B. Van Itallie, editors. John Libbey \& Co. Ltd., London. 163-170.

38. Kissebah, A. H., N. Vydelingum, R. Murray, D. J. Evans, A. J. Hartz, R. K. Kalkhoff, and P. W. Adams. 1982. Relation of body fat distribution to metabolic complications of obesity. J. Clin. Endocrinol. Metab. 54:254-260.

39. Glass, A. R., R. S. Swerdloff, G. A. Bray, W. T. Dahms, and R. L. Atkinson. 1977. Low serum testosterone and sex-hormone-binding-globulin in massively obese men. J. Clin. Endocrinol. Metab. 45:1211-1219.

40. Kley, H. K., T. Deselaers, H. Peerenboom, and H. L. Kruskemper. 1980. Enhanced conversion of androstenedione to estrogens in obese males. J. Clin. Endocrinol. Metab. 51:1128-1132.

41. Schindler, A. R., A. Ebert, and E. Friedrich. 1972. Conversion of androstenedione to estrone in human fat tissue. J. Clin. Endocrinol. Metab. 35:627-630.

42. Longcope, C., J. H. Pratt, S. H. Schneider, and S. E. Fineberg. 1978. Aromatization of androgens by muscle and adipose tissue in vivo. J. Clin. Endocrinol. Metab. 46:146-151.

43. Kley, H. K., P. Edelman, and H. L. Kruskemper. 1980. Relationship of plasma sex hormones to different parameters of obesity in male subjects. Metab. Clin. Exp. 29:1041-1045.

44. Hamalainen, E., H. Adlercreutz, C. Ehnholm, and C. Puska. 1986. Relationship of serum lipoproteins and apoproteins to sex hormones and to the binding capacity of sex-hormone-binding-globulin in health Finnish men. Metab. Clin. Exp. 35:535-541. 